\title{
Evaluating Central Aortic Blood Pressures in a Tertiary South Indian Hospital
}

\author{
Hemamalini Padma Kavirayani ${ }^{1}$ \\ ${ }^{1}$ Department of Cardiology, King George Hospital, Andhra Medical \\ College, Visakhapatnam, India
}

Indian J Cardiovasc Dis Women-WINCARS 2017;2:67-71

\author{
Address for correspondence Hemamalini Padma Kavirayani, DM, \\ Department of Cardiology, Andhra Medical College, \\ King George Hospital, Visakhapatnam, 530002, India \\ (e-mail: hemamailini@gmail.com).
}

Abstract

Keywords

- central aortic blood pressure

- peripheral blood pressure

- aortic diastolic blood pressure

- peripheral diastolic blood pressure
Background Of late, central aortic blood pressure (CABP) has emerged as a better parameter than peripheral blood pressure (BP) in the diagnosis of cardiovascular events. Advent of new technologies has facilitated the calculation of CABP from machinederived peripheral BP. In this study, the author determined the differences between peripheral BP measured manually or by machine and machine-derived CABP and examined whether this difference is stable even after categorizing the sample pool based on sex, hypertension, diabetes status, and $\beta$-blocker use.

Materials and Methods A total of 83 patients (both male and female) who attended the cardiology outpatient department were enrolled in the study. BP was recorded both manually and using Mobil-O-Graph pulse wave analyzer (ARC Solver) in the patient's sitting posture. The author compared the derived central, manual, and devicemeasured BP among the patient samples and assessed whether sex, hypertension, diabetes, and $\beta$-blocker use influence these differences.

Results Among the study population, 28 were females and 55 were males; patients' mean age was $59.97 \pm 12.15$ years. The mean peripheral systolic BP (SBP) and diastolic BP (DBP) measured manually were127.55 \pm 20.15 and $79.73 \pm 9.57 \mathrm{~mm} \mathrm{Hg}$, respectively. Similar measurements recorded by the device were $129.68 \pm 19.93$ and 78.92 \pm 13.48 , respectively. The derived mean central aortic SBP and DBP was $117.69 \pm 17.78$ and $80.15 \pm 13.71$, respectively. Statistically significant difference in the manual and central aortic SBP $(9.85 \pm 11.16 ; p<0.0001)$ was observed. This difference was significant irrespective of sex, hypertension, diabetes status, and $\beta$-blocker use. Similarly, difference between machine-derived peripheral SBP and central aortic SBP was statistically significant $(p<0.0001)$. However, the difference in manual and central aortic DBP was nonsignificant $(p=0.6976)$. Interestingly, a small $(-1.34 \pm 2.28)$ but statistically significant difference $(p<0.0001)$ between machine-derived peripheral DBP and central aortic DBP was observed. Further analysis to find out effect of $\beta$-blocker use on $C A B P$ revealed that the central aortic SBP is slightly, but statistically significantly, lower in $\beta$-blocker users $(117.7 \pm 17.71 ; p<0.0001)$ than that of $\beta$-blocker nonusers $(118.9 \pm 18.37 ; p<0.0001)$.

Conclusion Central aortic systolic pressure is statistically significantly lower than the manually recorded peripheral SBP irrespective of sex, hypertension, diabetes status, and $\beta$-blocker use. A small but significant difference was observed between machine-derived peripheral DBP and central aortic DBP. Patients using $\beta$-blockers were observed to have marginally lower CABP values than those who are not using them.
DOI https://doi.org/ $10.1055 / \mathrm{s}-0038-1622961$
Copyright @2017 Women in Cardiology and Related Sciences
License terms

(이 (1) $\Theta \circledast$ 


\section{Introduction}

The link between high blood pressure (BP) and increased cardiovascular risk has been established from a large body of data obtained with conventional (brachial cuff) sphygmomanometer measurements. Indeed, brachial BP parameters are reasonably predictive of cardiovascular morbidity and mortality. ${ }^{1}$ However, brachial BP may not completely reflect aortic degenerative changes that characterize the pathogenesis of cardiovascular disease. ${ }^{2,3}$

There is evolving evidence showing that different antihypertensive drugs with similar effects on brachial BP may have diverse effects on central aortic pressure (CAP). CAPs are influenced considerably by vascular endothelial function ${ }^{4,5}$ and provide a more accurate reflection of arterial hemodynamics. Additionally, CAP represents the BP actually perceived by the heart and brain and is therefore predictive of cardiovascular outcomes. ${ }^{6} \mathrm{BP}$ consists of a steady component (mean arterial pressure) and a pulsatile component (pulse pressure). ${ }^{7}$ The pulse pressure component, which is defined as the difference between brachial systolic blood pressure (SBP) and diastolic blood pressure (DBP), has been emphasized as a surrogate marker of large artery stiffness and a possible predictor of coronary heart disease risk independent of mean arterial pressure. ${ }^{8}$ There is a disparity, however, between central and peripheral pulse pressure due to a rise in SBP, ${ }^{9}$ which has been attributed, in part, to conventional cardiovascular risk factors such as hypercholesterolemia, ${ }^{10}$ smoking, ${ }^{11}$ and metabolic syndrome. ${ }^{12}$ This disparity diminishes with age due to aortic stiffening, for which an increase in pulse wave velocity is a surrogate. ${ }^{13}$ Biophysically, CAP is determined by two major factors: cardiac output and peripheral vascular resistance. ${ }^{4,14}$ Thus, CAP should give a more accurate reflection of the hemodynamic burden on the ejecting left ventricle, coronary, and cerebral vasculature, and theoretically may correspond more closely to cardiovascular events than to brachial BP.

\section{Materials and Methods}

A total of 83 patients (both male and female) who attended the cardiology outpatient department were enrolled in the study. BP was recorded both manually and using MobilO-Graph pulse wave analyzer (ARC Solver; I.E.M. GmbH) in patient's sitting posture. The author has compared the derived central, manual, and device-measured BP among the patient samples and assessed whether gender, hypertension, diabetes, and $\beta$-blocker use influence these differences (-Table $\mathbf{1}$ ).

\section{Results}

Among the study population, 28 were females and 55 were males; patients' mean age was $59.97 \pm 12.15$ years. The mean peripheral SBP and DBP measured manually were 127.55 \pm 20.15 and $79.73 \pm 9.57 \mathrm{~mm} \mathrm{Hg}$, respectively. Similar measurements recorded by the device were $129.68 \pm 19.93$ and $78.92 \pm 13.48$, respectively. The derived mean central aortic SBP and DBP was $117.69 \pm 17.78$ and $80.15 \pm 13.71$, respectively. Statistically significant difference in the manual and central aortic SBP $(9.85 \pm 11.16 ; p<0.0001)$ was observed. This difference was significant irrespective of sex, hypertension, diabetes status, and $\beta$-blocker use. Similarly, difference between machine-derived peripheral SBP and central aortic SBP was statistically significant $(p<0.0001)$. However, the difference in manual and central aortic DBP was nonsignificant $(p=0.6976)$. Interestingly, a small $(-1.34 \pm 2.28)$ but statistically significant difference $(p<0.0001)$ between machine-derived peripheral DBP and central aortic DBP was observed. Further analysis to find out effect of $\beta$-blocker use on central aortic blood pressure (CABP) revealed that the central aortic SBP is slightly, but statistically significantly, lower in $\beta$-blocker users $(117.7 \pm 17.71 ; p<0.0001)$ than that of $\beta$-blocker nonusers (118.9 $\pm 18.37 ; p<0.0001)$.

\section{Discussion}

Hypertension, a condition of elevated arterial BP conventionally diagnosed by brachial cuff sphygmomanometry, is associated with increased risk of cardiovascular mortality and morbidity and end-organ damage. However, the marked differences in pulse pressure between the central aorta and peripheral limbs suggest that effects of peak values of arterial BP (e.g., SBP) on centrally located organs (heart, brain,

Table 1 Comparison of BP measured by different methods

\begin{tabular}{|c|c|c|c|c|c|c|}
\hline S. no. & Method 1 & Mean \pm SD & Method 2 & Mean \pm SD & $p$ Value & Remarks \\
\hline 1 & Manual SBP & $126.83 \pm 19.37$ & $\begin{array}{l}\text { Machine-derived } \\
\text { central aortic SBP }\end{array}$ & $117.7 \pm 17.79$ & $<0.0001$ & $\begin{array}{l}\text { Statistically } \\
\text { significant }\end{array}$ \\
\hline 2 & Manual DBP & $79.73 \pm 9.58$ & $\begin{array}{l}\text { Machine-derived } \\
\text { central aortic DBP }\end{array}$ & $80.16 \pm 13.72$ & 0.6976 & $\begin{array}{l}\text { Not statistically } \\
\text { significant }\end{array}$ \\
\hline 3 & Manual SBP & $126.83 \pm 19.37$ & $\begin{array}{l}\text { Machine-derived } \\
\text { brachial SBP }\end{array}$ & $129.69 \pm 19.94$ & 0.0182 & $\begin{array}{l}\text { Statistically } \\
\text { significant }\end{array}$ \\
\hline 4 & Manual DBP & $79.73 \pm 9.58$ & $\begin{array}{l}\text { Machine-derived } \\
\text { brachial DBP }\end{array}$ & $78.93 \pm 13.49$ & 0.5187 & $\begin{array}{l}\text { Not statistically } \\
\text { significant }\end{array}$ \\
\hline 5 & $\begin{array}{l}\text { Machine-derived } \\
\text { brachial SBP }\end{array}$ & $129.69 \pm 19.94$ & $\begin{array}{l}\text { Machine-derived } \\
\text { central aortic SBP }\end{array}$ & $117.7 \pm 17.79$ & $<0.0001$ & $\begin{array}{l}\text { Statistically } \\
\text { significant }\end{array}$ \\
\hline 6 & $\begin{array}{l}\text { Machine-derived } \\
\text { brachial DBP }\end{array}$ & $78.93 \pm 13.49$ & $\begin{array}{l}\text { Machine-derived } \\
\text { central aortic DBP }\end{array}$ & $80.16 \pm 13.72$ & $<0.0001$ & $\begin{array}{l}\text { Statistically } \\
\text { significant }\end{array}$ \\
\hline
\end{tabular}

Abbreviations: BP, blood pressure; DBP, diastolic blood pressure; SBP, systolic blood pressure; SD, standard deviation. 
kidney) may not be accurately assessed using peripheral measurements.

Early studies showed substantial difference in the effects of sublingual nitroglycerin on peripheral and central (carotid) pulse pressures: in some cases, central SBP decreasing $20 \mathrm{~mm} \mathrm{Hg}$ or less with little or no effect on brachial or radial SBP. There were, however, marked changes in the pulse wave form. The relationship between central aortic and radial pressure waves, quantified in terms of a mathematical transfer function, has been validated to be applicable across a large range of physiological pressures. The use of this noninvasive technique (and other variations, including other forms of analysis of the radial pulse or direct registration of the carotid pulse) has facilitated a large number of studies highlighting the differential effects of antihypertensive therapy on central aortic SBP for similar values of brachial cuff SBP.

Seminal study by McEniery et al 5 in more than 10000 patients demonstrated a substantial overlap of central and brachial BP between categories of hypertension. Approximately $32 \%$ of men and $10 \%$ of women who would be considered to have normal brachial SBP (and therefore, not treated) would be classified as having stage 1 hypertension based on equivalent central aortic SBP. Indeed, the implications of these findings suggested a possible sign of a paradigm shift in the management and treatment of hypertension as a significant cardiovascular risk. ${ }^{6}$ Subsequent studies produced additional evidence of the possibility of added value of CABP. In the assessment of modern combination therapies, the amlodipine-valsartan combination was shown to decrease central aortic SBP to a greater extent than the amlodipine-atenolol combination for a similar effect on brachial SBP. Central pulse pressure has also been shown to be superior to ambulatory BP in the prediction of all-cause and cardiovascular mortality. ${ }^{8}$ However, notwithstanding these and other studies, there is still insufficient evidence for CABP to be integrated in guidelines for treatment and management of hypertension. The study by Sharman et $\mathrm{al}^{15}$ in this issue of hypertension addresses the use of CABP as an additional measure in the management of hypertension. However, in contrast to other studies that perform a comparison of effects of treatments or risk categories, this study uses CABP as a guide for treatment. Although conducted in a relatively small population (286 hypertensive patients), the study is particular in both the design and the relevance of the demonstration of the added value of CABP. It is a prospective, randomized, open-label, blinded end point (PROBE) study in which patients were randomized to treatment decisions that were guided by best-practice usual care for BP ( $n=142$; using office BP, home BP, and 24-hour ambulatory BP) or the addition of a CABP intervention $(n=144)$ where CABP was measured using radial applanation tonometry (SphygmoCor, AtCor Medical Pty Ltd.). The study duration was 12 months, and therapy was reviewed at intervals of 3 months. A key element of the study is the guidelines and recommendations given to the treating practitioners for titration of therapy. The five recommendation scenarios describe the combination of CABP with the other BP measures (office, home, 24-hour ambulatory $\mathrm{BP}$ ) to increase, maintain, or decrease the therapy. In addition to reaching the target $\mathrm{BP}$ using the conventional brachial cuff values, the study contains three specific outcome measures: the World Health Organization (WHO) standard metric for quantity of medication (daily defined dose [DDD]), quality-of-life questionnaire, and left ventricular (LV) mass obtained by three-dimensional echocardiography. The study shows an impressively high adherence ( $92 \%$ ) by practitioners and patients to the recommendations, with an improvement of quality of life in both the groups. The usual care BP group showed no change in DDD, whereas the CABP group showed a progressive decrease in DDD at each of the 3-month intervals for a similar level of brachial cuff BP between the groups. The study demonstrates for the first time that if CABP is included in the measurement and is also used as a guide to titrate therapy, a similar target brachial BP can be achieved with a reduction in medication as quantified by DDD. In addition, there was a much higher proportion of patients in the CABP group that ceased medication altogether (16\%) compared with the usual care group (2\%). All groups showed no statistical difference in LV mass, but the CABP group showed a trend for a reduction, whereas the usual care group showed a trend for an increase. Although the effects were relatively small (change in LV mass of $\approx 0.26 \mathrm{~g}$ ), the statistical significance is borderline $(p=0.079)$.

There was also no difference in aortic pulse wave velocity in all the groups, although this is not surprising, given that the values of mean and diastolic pressures were similar in both groups. Although demonstrating a potential for added value of CABP in guiding hypertension management, the study by Sharman et $\mathrm{al}^{15}$ raises several questions on the underlying significance of the results and the conclusions reached. One issue is the effect of heart rate on the relationship between central and radial pulse pressure, ${ }^{1,3}$ as was seen in the studies assessing the effects of $\beta$-adrenergic blockade (atenolol) as a hypertensive treatment, ${ }^{4}$ in which central aortic systolic pressure was shown to be relatively higher than the amlodipine group for similar brachial systolic pressure. However, this was not a significant concern in the study by Sharman et $\mathrm{al}^{15}$ because only $\approx 7 \%$ of patients in the whole cohort was on $\beta$-blockers, and there was no difference in heart rate among the groups. The other issue is the effects of age on the normal $\mathrm{BP}$ values. For both brachial and CABP, the range of normal values used in the study increased with age. The author makes the valid point that, indeed, a decrease in DDD could also be achieved simply because the target BP values would increase with age, hence requiring reduced medication. However, a plausible explanation for this is offered, which is supported by the findings of the effects of CABP-guided therapy on LV mass index. If the marked reduction in DDD in the CABP group occurred because of a fortuitous coincidence of the effects of age caused by increased target values, there would be an expected rise in LV mass index, presumably because of an attenuated effect of treatment on BP. However, the study found the opposite: a reduced DDD in the CABP group was associated with a trend for reduced LV mass index. From this study results, the author makes the potentially powerful assertion that antihypertensive therapy guided by CABP provides a more appropriate form of treatment. Thus, 
the suggestion is that this may provide the groundwork for the introduction of CABP in the clinical management of hypertension. However, an apparent limitation of the study is that the use of CABP would seem to be more effective in those with a large difference between central aortic and brachial systolic pressure. This is predominantly seen in younger individuals because the largest difference in central and peripheral systolic pressure is seen in age groups below the fifth decade of age, whereas the most hypertensive individuals are older than 50 years. However, a difference of $\approx 11 \mathrm{~mm}$ $\mathrm{Hg}$ has been found to persist till the age of $80,{ }^{5}$ which was similar to the mean difference found in the study by Sharman et $\mathrm{al}^{15}$ for a mean age of 64 years. This indicates that the procedure could still be of benefit in the elderly, although future studies would need to produce data on threshold differences in which CABP would not improve treatment.

The 2013 European Society of Hypertension/European Society of Cardiology Guidelines for the management of arterial hypertension ${ }^{9}$ state that although the measurement of CABP is of interest in terms of analyses for elucidating mechanisms related to pathophysiology, pharmacology, and therapeutics, further investigations are needed before CABP can be recommended for routine clinical use (with the only possible exception being isolated systolic hypertension in youth). Indeed, trials are required to assess hard end points, where patients are followed up for a longer period (of the order of 5 years, similar to many other intervention studies). In addition, the design should be expanded in which CABP is measured in all patients, but in which one group is assessed by measurements of brachial cuff pressure but blind to the results of central $\mathrm{BP}$ and the other groups are guided by the results of central BP but blind to the results of brachial BP. The study by Sharman et $\mathrm{al}^{15}$ provides the basis for these further investigations. It provides clear evidence that the addition of CABP can improve management of hypertension. However, although the intention of the PROBE design is to provide results that would be applicable to the real-world methods used by practitioners to treat and manage hypertension, it is not known to what extent this design contributed to any underlying bias among practitioners who were using the additional and novel measurement of CABP. In this and other studies, CABP was estimated using applanation tonometry. The requirements for operator training and additional time required for the procedure limit the practical use in the clinical setting, and to date CABP has been essentially an informative research tool. However, with new devices that are able to measure CABP using the conventional brachial cuff, future studies will have the capacity to be expanded to include both office and 24-hour CABP measurements. The overlap between brachial and central BP found in the study by McEniery et $\mathrm{al}^{9}$ among categories of hypertension implies that based simply on the brachial cuff BP, but in reference to the effects of CABP on end-organ damage, there are some individuals who should be treated and who are not and others who are on treatment and perhaps might not require it. The paradigm shift that was suggested by these observations ${ }^{6}$ would now seem to be supported by novel (although limited) confirmatory evidence from the study by Sharman et al, ${ }^{15}$ in which use of CABP to guide therapy is shown to provide improvement in the efficacy of management of hypertension through reduction in medication, as well as an additional effect with the possibility of reducing LV mass for similar values of brachial cuff SBP. The logical consequence of the confirmation of this paradigm shift is a potential pathway for the consideration of inclusion of CABP in the clinical management of hypertension.

\section{Conclusion}

Evidence is mounting to suggest that central pressures more closely correlate with measures of cardiovascular risk than brachial pressure and that central pressures independently predict future cardiovascular events and responses to antihypertensive therapy. Disparities between CAP and brachial BP measurements pose a challenge to the guidelines for the management of hypertension, as these are usually determined by conventional brachial cuff measurements. As the cost of required equipment comes down and additional persuasive evidence of its superiority over peripheral assessments becomes more abundant, assessing CAP may be the next important advance in the future clinical management of hypertension.

\section{References}

1 Lewington S, Clarke R, Qizilbash N, Peto R, Collins R; Prospective Studies Collaboration. Age-specific relevance of usual blood pressure to vascular mortality: a meta-analysis of individual data for one million adults in 61 prospective studies. Lancet 2002;360(9349):1903-1913

2 Agabiti-Rosei E, Mancia G, O’Rourke MF, et al. Central blood pressure measurements and antihypertensive therapy: a consensus document. Hypertension 2007;50(1):154-160

3 Protogerou AD, Papaioannou TG, Blacher J, Papamichael CM, Lekakis JP, Safar ME. Central blood pressures: do we need them in the management of cardiovascular disease? Is it a feasible therapeutic target? J Hypertens 2007;25(2):265-272

4 Weber T, Auer J, O'Rourke MF, et al. Arterial stiffness, wave reflections, and the risk of coronary artery disease. Circulation 2004;109(2):184-189

5 O'Rourke MF, Seward JB. Central arterial pressure and arterial pressure pulse: new views entering the second century after Korotkov. Mayo Clin Proc 2006;81(8):1057-1068

6 Hirata K, Vlachopoulos C, Adji A, O’Rourke MF. Benefits from angiotensin-converting enzyme inhibitor 'beyond blood pressure lowering': beyond blood pressure or beyond the brachial artery? J Hypertens 2005;23(3):551-556

7 Safar ME, Boudier HS. Vascular development, pulse pressure, and the mechanisms of hypertension. Hypertension 2005; 46(1):205-209

8 Franklin SS, Khan SA, Wong ND, Larson MG, Levy D. Is pulse pressure useful in predicting risk for coronary heart disease? The Framingham heart study. Circulation 1999;100(4):354-360

9 McEniery CM, Yasmin, McDonnell B, et al; Anglo-Cardiff Collaborative Trial Investigators. Central pressure: variability and impact of cardiovascular risk factors: the Anglo-Cardiff Collaborative Trial II. Hypertension 2008;51(6):1476-1482 
10 Wilkinson IB, Prasad K, Hall IR, et al. Increased central pulse pressure and augmentation index in subjects with hypercholesterolemia. J Am Coll Cardiol 2002;39(6):1005-1011

11 Mahmud A, Feely J. Effect of smoking on arterial stiffness and pulse pressure amplification. Hypertension 2003; 41(1):183-187

12 Protogerou AD, Blacher J, Mavrikakis M, Lekakis J, Safar ME. Increased pulse pressure amplification in treated hypertensive subjects with metabolic syndrome. Am J Hypertens 2007;20(2):127-133

13 Williams B, Lacy PS, Thom SM, et al; CAFE Investigators; Anglo-Scandinavian Cardiac Outcomes Trial Investigators;
CAFE Steering Committee and Writing Committee. Differential impact of blood pressure-lowering drugs on central aortic pressure and clinical outcomes: principal results of the Conduit Artery Function Evaluation (CAFE) study. Circulation 2006;113(9):1213-1225

14 Izzo JL Jr. Arterial stiffness and the systolic hypertension syndrome. Curr Opin Cardiol 2004;19(4):341-352

15 Sharman JE, Marwick TH, Gilroy D, et al. Randomized trial of guiding hypertension management using central aortic blood pressure compared with best-practice care. Principal findings of the BP GUIDE Study. Hypertension 2013; 62(6):1138-45 